\title{
Perfil algológico del paciente con enfermedad del sistema musculoesquelético
}

\section{Algological profile of the patient with musculoskeletal system disease}

\author{
Dra. Yadira Irlette Martínez-Ruiz,* Dra. Evelyn Judith Cruz-Nocelo, ${ }^{\ddagger}$ \\ Dra. María Concepción Serratos-Vázquez
}

Citar como: Martínez-Ruiz YI, Cruz-Nocelo EJ, Serratos-Vázquez MC. Perfil algológico del paciente con enfermedad del sistema musculoesquelético. Rev Mex Anestesiol. 2021; 44 (3): 166-172. https://dx.doi.org/10.35366/99662

RESUMEN. Introducción: Se estima que del total de personas que refieren dolor crónico, uno de cada cuatro presenta dolor de origen musculoesquelético. El objetivo del estudio fue analizar el perfil demográfico y clínico del paciente con patología musculoesquelética que acude por primera vez a un centro especializado en tratamiento del dolor. Material y métodos: Se realizó un estudio transversal, descriptivo y retrospectivo que consistió en la revisión de la historia clínica algológica de los expedientes archivados del 2009 al 2017 del Centro Interdisciplinario para el Estudio y Tratamiento del Dolor y Cuidados Paliativos de la UMAE «Dr. Victorio de la Fuente Narváez». Resultados: Se incluyeron 4,518 expedientes. La mediana de la edad de los pacientes fue de 59 años (RIQ 47:72), consultando con mayor frecuencia el género femenino (62\%). El dolor crónico se presentó en $83 \%$ de los pacientes con una intensidad media valorada por la escala visual analógica (EVA) de $6( \pm 2.4)$. El síndrome doloroso mixto (somático y neuropático) se presentó con mayor frecuencia (41\%). Conclusiones: La progresión de las enfermedades crónico degenerativas sobre el sistema musculoesquelético impacta en el origen y evolución de dolor crónico. El momento en el que los pacientes son remitidos a un centro especializado y reciben tratamiento específico del dolor podría modificar la evolución y consecuencias de éste.

ABSTRACT. Introduction: It is estimated that of the total number of people who report chronic pain, one in every four presents pain of musculoskeletal origin. The objective of the study was to analyze the demographic and clinical profile of the patient with musculoskeletal pathology who visits a specialized pain treatment center for the first time. Material and methods: A cross-sectional, descriptive and retrospective study consisting of the review of the clinical history of the records archived from 2009 to 2017 of the Interdisciplinary Center for the Study and Treatment of Pain and Palliative Care of the UMAE «Dr. Victorio de la Fuente Narváez». Results: 4,518 records were included. The median age of patients was 59 years (IQR 47:72), with more frequent consultation for the female gender (62\%). Chronic pain occurred in $83 \%$ of patients with an intensity assessed by the average visual analog scale (VAS) of 6 ( \pm 2.4 ). Mixed pain syndrome (somatic and neuropathic) occurred more frequently (41\%). Conclusions: The progression of chronic degenerative diseases on the musculoskeletal system impacts on the origin and evolution of chronic pain. The moment in which patients are referred to a clinic of pain and receive specific treatment could modify its evolution and consequences.

\section{INTRODUCCIÓN}

L a Organización Mundial de la Salud (OMS) reconoce al dolor crónico como un problema de salud pública ${ }^{(1)}$. A nivel internacional, se ha estimado que afecta del 25 al $29 \%$ de la población general ${ }^{(2)}$ representando del 15 al 20\% de las consultas médicas ${ }^{(3)}$. En México, es posible que poco más de 28 millones de habitantes padezcan este tipo de dolor ${ }^{(2)}$.

El dolor crónico se ha reconocido como aquel dolor que persiste más allá del tiempo de curación normal, carece de la función de advertencia aguda de la nocicepción fisiológica y es definido como un dolor persistente o recurrente con duración mayor a tres meses ${ }^{(3)}$. Se estima que del total de personas que refieren dolor crónico, uno de cada cuatro presenta dolor de origen musculoesquelético ${ }^{(4)}$.

Numerosos estudios han documentado el manejo del dolor crónico en una variedad de contextos con resultados variables; sin embargo, los mejores resultados se han observado en las clínicas multidisciplinarias de dolor. Lamentablemente, en la actualidad estos servicios

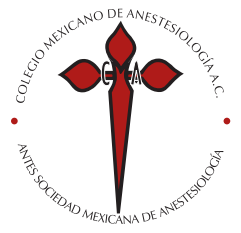

Palabras clave:

Dolor crónico, clínica del dolor, dolor musculoesquelético, dolor lumbar.

Keywords:

Chronic pain, pain clinics, musculoskeletal pain, low back pain.

* Servicio de Anestesiología del Hospital de Traumatología.

‡ Centro Interdisciplinario para el Estudio y Tratamiento del Dolor y Cuidados Paliativos.

Unidad Médica de Alta Especialidad «Dr. Victorio de la Fuente Narváez», Instituto Mexicano del Seguro Social. Ciudad de México, México.

Correspondencia:

Dra. Evelyn Judith Cruz-Nocelo Av. Pavo Real ext. 60, int. 21, Col. Las Alamedas, 52970, Atizapán, Estado de México, México.

Teléfono: 55 1798-2170 / 55

5747-3500, Ext. 25555 y 25556

E-mail: eve_412@hotmail.com,

Recibido: 25-10-2018

Aceptado: 05-03-2021

Abreviaturas:

CIETD-CP = Centro

Interdisciplinario para el

Estudio y Tratamiento del

Dolor y Cuidados Paliativos.

HGZ = Hospital General de Zona.

ROA = Rescate osteoarticular.

SCAR = Servicio de Cirugía

Articular Reconstructiva.

UMAE = Unidad Médica

de Alta Especialidad.

$\mathrm{UMF}=$ Unidad de

Medicina Familiar.

HGZ = Hospital General de Zona. 
no están disponibles para la totalidad de la población; en consecuencia, la mayor parte de la atención médica del dolor crónico se brinda en primer nivel ${ }^{(5)}$.

La Unidad Médica de Alta Especialidad (UMAE) «Dr. Victorio de la Fuente Narváez» del Instituto Mexicano del Seguro Social (IMSS) está constituida por tres hospitales (el Hospital de Ortopedia, el Hospital de Traumatología y la Unidad de Medicina Física y Rehabilitación Norte) dirigidos al estudio y tratamiento de los pacientes con patología neuromusculoesquelética en fase aguda, crónica-degenerativa y en fase de secuela, otorgando al día un promedio de 840 consultas de especialidad. En el 2009 comenzó a funcionar la Clínica del Dolor de esta unidad para posteriormente establecerse como el Centro Interdisciplinario para el Estudio y Tratamiento del Dolor y Cuidados Paliativos (CIETD-CP). El objetivo de este estudio fue identificar y analizar el perfil demográfico y clínico de los pacientes que acuden por primera vez a este centro.

\section{MATERIAL Y MÉTODOS}

Previa aprobación por el comité de ética local con el número de registro R-2017-3401-15, se realizó un estudio transversal, descriptivo y retrospectivo que consistió en la revisión de los expedientes clínicos de la consulta externa de primera vez del CIETD-CP de la UMAE «Dr. Victorio de la Fuente Narváez» durante un período de ocho años (del 31 de marzo de 2009 al 31 de agosto de 2017) para obtener los datos de la historia clínica algológica de cada uno de ellos. Las variables demográficas analizadas fueron edad y género, las variables clínicas fueron: servicio de referencia, comorbilidades, tratamiento previo, diagnóstico algológico y tratamiento inicial del CIETD-CP. Las variables algológicas fueron: origen del dolor, tiempo del dolor, sitio de localización del dolor, temporalidad del dolor, causa del

\section{Edad de los pacientes del CIETD-CP}

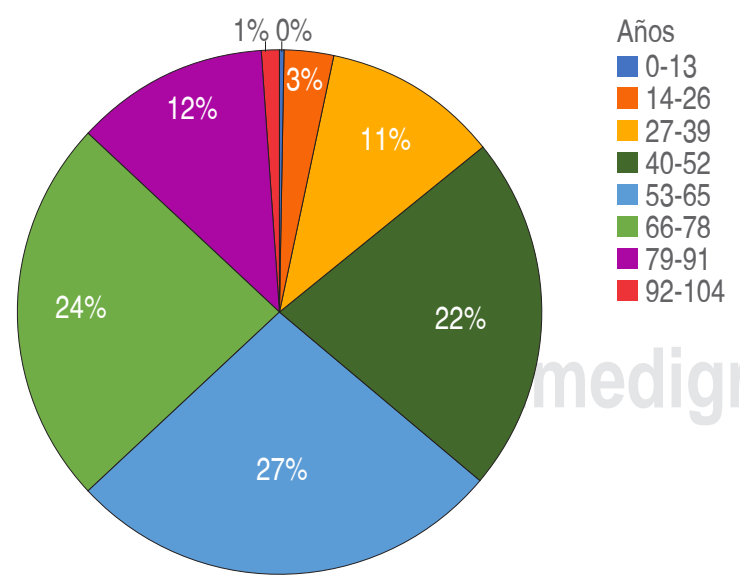

Figura 1: Gráfica de frecuencias relativas de la edad de los pacientes del CIETD-CP.

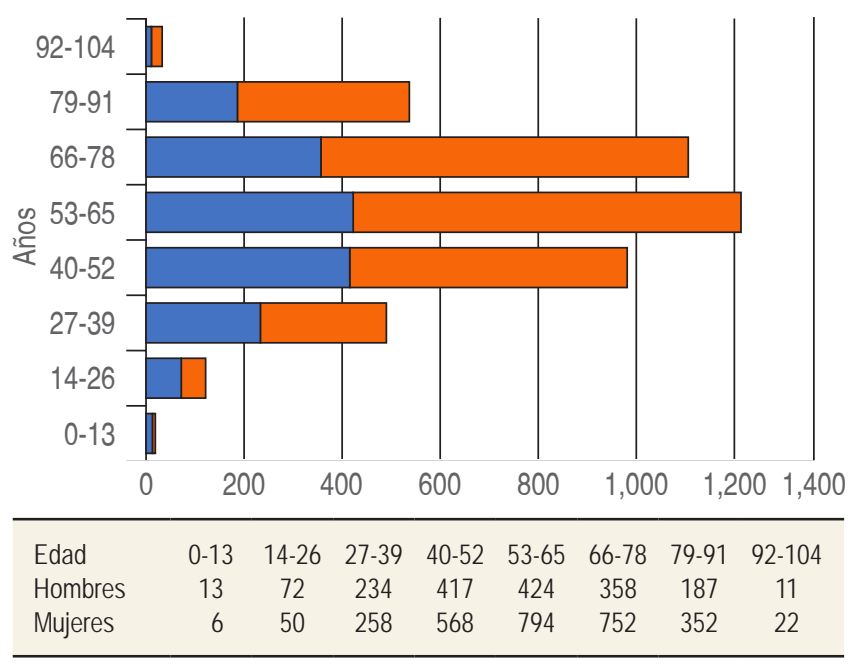

Figura 2: Gráfica comparativa de las frecuencias absolutas de la edad por género de los pacientes del CIETD-CP.

dolor, dinámica e intensidad del dolor. El análisis estadístico se realizó a través el programa Excel, para el análisis descriptivo las variables cuantitativas fueron presentadas con medidas de tendencia central (media o mediana) y de dispersión (desviación estándar o rango intercuartil). Asimismo, los resultados de las variables cualitativas fueron expresados en frecuencias absolutas y relativas.

\section{RESULTADOS}

Se obtuvo un total de 4,788 registros de consultas de primera vez, de los cuales 270 expedientes fueron descartados debido a información incompleta; por lo tanto, se incluyeron un total de 4,518 expedientes en el estudio. La mediana de la edad de los pacientes fue de 59 años (RIQ 47:72). Como se muestra en la Figura 1, el 48.8\% de los pacientes tuvieron rangos de edad entre los 40 y 65 años y el $24.6 \%$ entre los 66 y 78 años. Al realizar el análisis por género, observamos que el $62 \%$ de las consultas fueron otorgadas a pacientes del género femenino. En la Figura 2 se muestran las frecuencias absolutas de la edad de los pacientes valorados por género.

Dentro de las variables algológicas se estimó el tiempo desde el inicio del dolor hasta el momento en que el paciente fue valorado por primera vez por el CIETD-CP. La mediana de este tiempo fue de 12 meses (RIQ 6:36). Como se muestra en la Figura 3, el 51\% de los pacientes fue referido y valorado por el CIETD-CP dentro del primer año de evolución del dolor.

Respecto a la temporalidad, el dolor de tipo crónico se presentó en el 83\% de las consultas de primera vez y se categorizó en dolor crónico agudizado (43\%) y no agudizado (45\%). Mientras que el dolor agudo tuvo una prevalencia de $12 \%$. 
Por otra parte, en la Figura 4 se observan los resultados de la valoración de la intensidad del dolor por medio de dos escalas unidimensionales: 1) escala visual analógica (EVA) y 2) escala verbal analógica (EVERA). De acuerdo con la EVA, la intensidad del dolor fue evaluada en una media de $6( \pm 2.4)$. Con la EVERA se reportó dolor de intensidad moderada en el $43 \%(1,952)$ y severa en el $40 \%(1,795)$ de los pacientes.

La dinámica de presentación del dolor que se reportó con mayor frecuencia fue de tipo continuo fluctuante en el 74.5\% de los casos. Los sitios de localización anatómica del dolor se encuentran graficados en la Figura 5; los sitios referidos con mayor frecuencia fueron: dolor localizado en piernas con el $64 \%$, dolor lumbosacro con el 51\% y dolor en brazos con el $25.5 \%$. El 40\% de pacientes refirieron simultáneamente dolor localizado en región lumbosacra y en piernas.

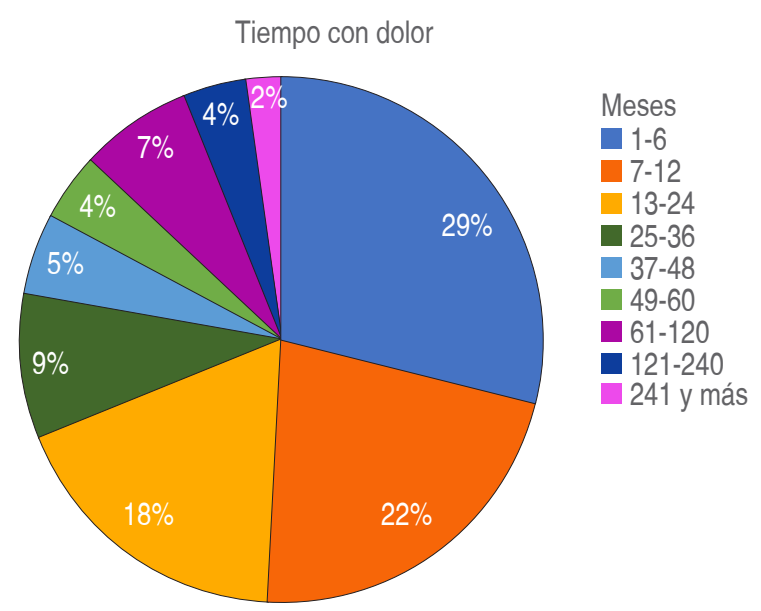

Figura 3: Representación gráfica del tiempo de evolución del dolor al momento de la primera valoración por el CIETD-CP. Mediana de 12 meses (RIQ 6:36).
En el CIETD-CP el 93\% $(4,185)$ de los pacientes consultados presentaron dolor de origen no oncológico. La causa del dolor fue clasificada en: enfermedad, traumatismo, posterior a procedimiento quirúrgico o alguna combinación de éstas; la enfermedad fue la causa más frecuente del dolor (48\%), seguida del traumatismo (26\%).

Las comorbilidades que refirieron los pacientes se muestran en la Figura 6, las enfermedades más frecuentes fueron de tipo crónico degenerativo, la hipertensión arterial en el 35.7\% $(1,614)$ de los pacientes y diabetes mellitus en el 20.7\% (935) fueron las más frecuentes. Sólo el 30\% $(1,369)$ de los pacientes no tuvieron comorbilidades.

Respecto al diagnóstico algológico, como se puede observar en la Tabla 1, el síndrome doloroso mixto (somático y neuropático) fue el que se presentó con más frecuencia (41\%), seguido del síndrome doloroso neuropático periférico (27\%) y del síndrome doloroso nociceptivo somático (21\%). En la Tabla 2 se muestran las frecuencias del servicio de referencia de los pacientes al CIETD-CP. El $42 \%$ de los pacientes fueron referidos por el Servicio de Columna. Mientras que el 28\% procedían de su Unidad de Medicina Familiar (UMF) del Hospital General de Zona (HGZ) o algún otro.

Respecto al tratamiento previo a su ingreso al CIETD$\mathrm{CP}$, el $45 \%$ ya tenían un esquema de tratamiento continuo o intermitente con uno o varios AINE (antiinflamatorio no esteroideo). Sólo el 18\% estaban empleando algún opioide al momento de la valoración. A su ingreso al CIETD-CP, en sólo 6\% de los casos se continuó o inició el tratamiento con AINE. El 89\% de los pacientes se trataron con opioides, el tramadol fue empleado en el 84\% de los casos. Asimismo, el paracetamol se prescribió en el 75\%. De esta manera, los opioides y el paracetamol fueron la base de los esquemas de tratamiento inicial del CIETD-CP. Los coadyuvantes analgésicos de mayor prescripción fueron los neuromoduladores, de los cuales los anticomiciales fueron los más frecuentes (74\%).
A

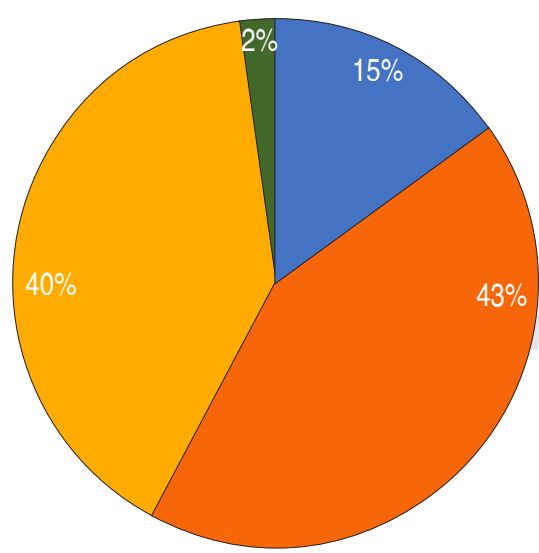

Leve

Moderado

Severo

Sin dolor

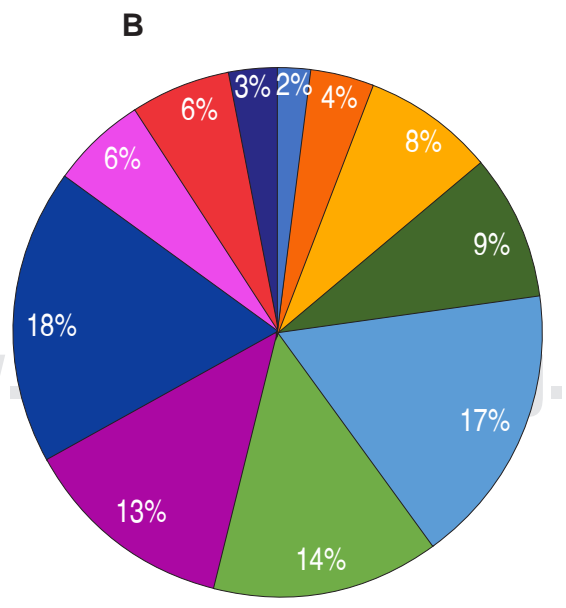

$1 \square 2 \square 3 \square 4 \square 5 \square 6 \square 7 \square 8 \square 9 \square 10 \square S D$
Figura 4:

A) Intensidad del dolor valorado con EVERA. B) Intensidad del dolor valorado con EVA. El 83\% expresaron un EVA correspondiente con un dolor moderado a severo. 


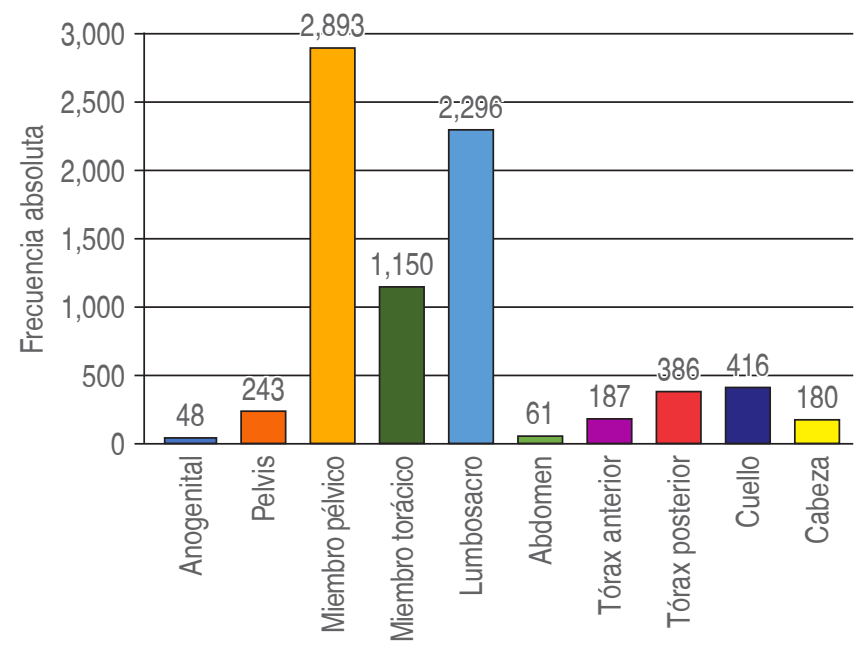

Figura 5: Gráfica de la frecuencia de los sitios anatómicos afectados por el dolor. El dolor localizado en piernas, el dolor lumbosacro y el dolor en brazos fueron los sitios de dolor referidos con mayor frecuencia.

\section{DISCUSIÓN}

En la actualidad, el dolor crónico puede considerarse como el tercer problema de salud más importante en nuestro entorno después de las enfermedades cardiovasculares y el cáncer. Éste tiene importantes repercusiones económicas, no sólo aquéllas asociadas con los costos médicos, quirúrgicos y farmacéuticos, sino también al ausentismo laboral, discapacidad y disminución de la calidad de vida ${ }^{(6)}$.

De acuerdo con los resultados del presente estudio, el dolor lumbosacro es uno de los que se presentaron con mayor frecuencia; además, el $40 \%$ de pacientes refirieron simultáneamente dolor localizado en la región lumbosacra $\mathrm{y}$ en piernas. Estudios previos sugieren que el dolor neuropático está presente en el 35 al 55\% de pacientes con dolor lumbosacro irradiado a miembros pélvicos ${ }^{(7)}$. Sin embargo, actualmente se ha sugerido que probablemente la lumbalgia con componente neuropático se podría presentar con mayor frecuencia. El dolor nociceptivo, el dolor neuropático periférico y el dolor de sensibilización central han sido sugeridos como mecanismos del dolor musculoesquelético, en el que cada clasificación se refiere a una presentación clínica del predominio de los mecanismos nociceptivos, neuropáticos periféricos o neuropáticos centrales, respectivamente ${ }^{(8)}$.

Asimismo, la lumbalgia es considerada como un importante problema de salud pública en todo el mundo. Se estima que la lumbalgia afecta hasta a un $84 \%$ de la población durante algún momento a lo largo de su vida. En el caso de lumbalgia crónica, se estima una prevalencia del 15 al 36\% ${ }^{(9)}$. De acuerdo con el Global Burden of Disease Study (2010), la lumbalgia genera más discapacidades que cualquier otra enfermedad en todo el mundo, representa el $10.7 \%$ del total de años vividos con discapacidad y ocupa el sexto lugar en cuanto a la carga global de enfermedad con 83 millones de años de vida ajustados a discapacidad ${ }^{(10)}$.

En México, la esperanza de vida saludable (sin discapacidad) se estima en 67.1 años en las mujeres y de 63.6 años en los hombres, lo que significa que las mujeres viven aproximadamente 11.3 años y los hombres 10 años de su vida (casi siempre la última etapa) con discapacidad ${ }^{(8)}$. De acuerdo con ese estudio, las causas asociadas principalmente a discapacidad fueron las enfermedades musculoesqueléticas (23\% en mujeres vs. 19\% en hombres) y trastornos mentales ( $23 \%$ en mujeres vs. $25 \%$ en hombres); dentro de estas causas destacaron los siguientes padecimientos: depresión mayor (8.3\% en mujeres vs. 5.5\% en hombres) y lumbalgia (6.7\% en mujeres vs. $8.4 \%$ en hombres) ${ }^{(11)}$. En los resultados del presente estudio, la lumbalgia se presentó en la mitad de los pacientes con dolor de origen musculoesquelético, la depresión estuvo asociada con lumbalgia sólo en el 14\%.

Por otra parte, se estima que la incidencia del dolor lumbar y del dolor crónico en general se incrementará debido a la clara transición epidemiológica por la que México atraviesa; se calcula que en el 2030 habrá casi 43 adultos mayores por cada 100 jóvenes ${ }^{(12)}$. De esta manera, simultáneamente se ha observado un incremento progresivo de las enfermedades no transmisibles como la hipertensión arterial sistémica, diabetes mellitus, obesidad y cáncer, que en el país son las primeras causas de morbilidad y mortalidad. Esto también lo pudimos confirmar en el presente estudio, en el cual los resultados reflejaron que las tres comorbilidades principales registradas fueron hipertensión arterial, diabetes mellitus y la obesidad; de manera similar, con el incremento en la esperanza de vida también se han incrementado las afecciones dolorosas asociadas con la enfermedad degenerativa musculoesquelética

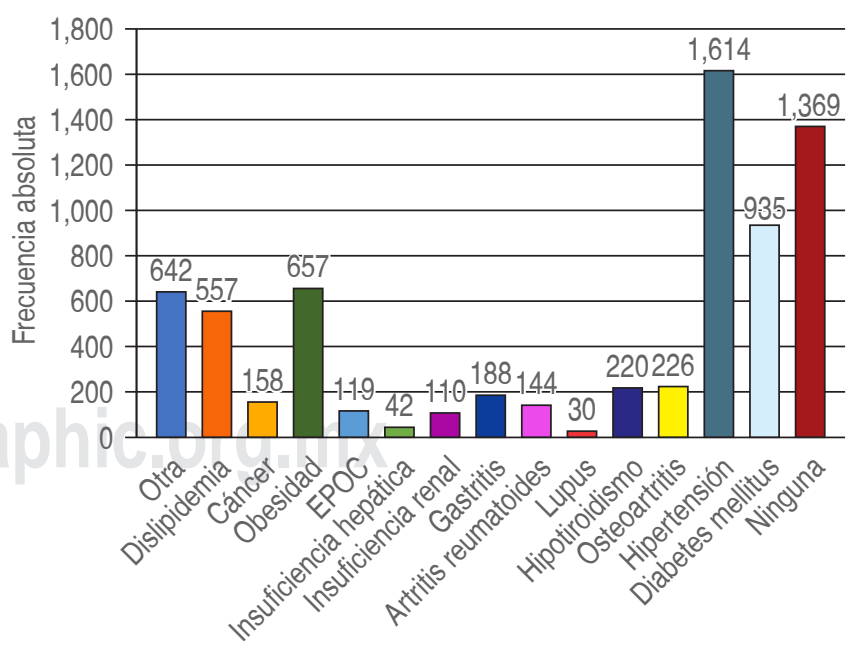

Figura 6: Gráfica de comorbilidades de los pacientes de primera vez del CIETD-CP. 
(p. ej: artritis reumatoide y osteoporosis) y a algunas neuropatías (p. ej: neuralgia postherpética y neuropatías diabéticas) ${ }^{(13)}$.

El dolor crónico tiene un fuerte impacto social y al estar frecuentemente asociado con otras enfermedades crónicas representa una carga para los sistemas de salud y la economía. Es un problema de salud específico con características claras de síntomas, incapacidad y problemas de salud mental que son en gran medida independientes de la enfermedad o lesión subyacente ${ }^{(14)}$. El dolor crónico de origen osteoarticular degenerativo o inflamatorio afecta a la salud física y psicológica de quien lo padece, con afectación multidimensional de la calidad de vida como son repercusiones sobre las actividades diarias, autonomía, empleo y bienestar económico ${ }^{(15)}$. Se ha descrito un vínculo entre la intensidad del dolor y la calidad de vida en pacientes con dolor crónico, lo que demuestra que cuanto más fuerte es la intensidad del dolor menor es la calidad de $\operatorname{vida}^{(16)}$. Debido a estas implicaciones, la referencia a una clínica multidisciplinaria especializada en el manejo del dolor debe considerarse una vez que las modalidades de tratamiento en las primeras semanas o meses después del inicio del dolor se han considerado infructuosas ${ }^{(17)}$. Sin embargo, en este estudio se evidencia que en el $50 \%$ de los pacientes la valoración del dolor por un centro especializado en el manejo del dolor es mayor a un año después del inicio de su dolor. Ciertamente, se sabe que un retardo en la evaluación y tratamiento del dolor crónico produce una disfunción progresiva de los sistemas opioides endógenos con la consiguiente disregulación en el sistema cardiovascular y en las vías descendentes inhibitorias del dolor. Esto podría ser un detonante de cambios complejos irreversibles del sistema nervioso central que evolucionan con el tiempo a las condiciones de dolor crónico. En la actualidad, existe evidencia creciente de la neuroplasticidad, particularmente en el sistema límbico, en el desarrollo de síndromes dolorosos patológicos ${ }^{(13)}$.

Por otra parte, en los resultados del presente estudio se observó la prescripción alta de AINE (45\%) por períodos prolongados por parte del primer y segundo nivel de atención médica, probablemente debido a su efectividad para el dolor leve a moderado y por no causar dependencia ni tolerancia; sin embargo, su problema se fundamenta en que poseen un efecto analgésico techo y múltiples efectos secundarios por

Tabla 1: Distribución de frecuencia del diagnóstico algológico.

\begin{tabular}{lrr} 
Síndrome doloroso & $\mathrm{n}$ & $\%$ \\
\hline Nociceptivo somático & 929 & 21 \\
Nociceptivo visceral & 42 & 1 \\
Neuropático periférico & 1,221 & 27 \\
Neuropático central & 395 & 9 \\
Mixto (somático y neuropático) & 1,848 & 41 \\
Otros & 83 & 2
\end{tabular}

Tabla 2: Distribución de frecuencia del servicio de referencia.

\begin{tabular}{lrr} 
Servicio de referencia & $\mathrm{n}$ & $\%$ \\
\hline Columna & 1,878 & 42 \\
Tumores/oncología & 135 & 3 \\
Rehabilitación & 124 & 3 \\
ROA, SCAR, artroscopía & 110 & 2 \\
Neurocirugía & 55 & 1 \\
Miembro torácico & 269 & 6 \\
Pelvis, cadera y acetábulo & 119 & 3 \\
Fémur y rodilla, pie y tobillo & 299 & 7 \\
UMF, HGZ, otro & 1,290 & 29 \\
Cirugía plástica reconstructiva & 137 & 3 \\
Quemados & 12 & 0 \\
Poliexpuestas & 82 & 2 \\
No especificado & 8 & 0 \\
\hline
\end{tabular}

ROA = rescate osteoarticular; SCAR = Servicio de Cirugía Articular Reconstructiva; UMF = Unidad de Medicina Familiar; HGZ = Hospital General de Zona.

consumo crónico ${ }^{(18)}$. Además, esto contrasta con lo publicado en los «parámetros de práctica para el manejo del dolor en México», en donde se recomienda que la administración de AINE no debe exceder de cinco días ${ }^{(19)}$.

En el tratamiento otorgado en la valoración inicial por la clínica del dolor se reflejó un alto uso de opioides como primera línea de tratamiento, principalmente del tramadol, opioide débil, para dolor de moderada a severa intensidad en el 83\% de los casos. En el año 2015, la comunidad científica sugirió una taxonomía racional basada tanto en los mecanismos del dolor como en las dianas moleculares de los analgésicos, estableciendo que, en lo que se refiere al dolor crónico, el dolor nociceptivo inflamatorio podría ser tratado con el objetivo de la reducción de la inflamación con esteroides o AINE, el dolor nociceptivo no inflamatorio con opioides y analgésicos no opioides, y el dolor neuropático con antidepresivos y antiepilépticos, incluyendo fármacos especíicos en determinadas situaciones clínicas reumatológicas ${ }^{(20)}$. Una concepción diferente a la de la escalera analgésica de la OMS permitiría tratar el dolor de acuerdo con la realidad clínica y evita el encasillamiento en una escalada de fármacos más potentes cuando no hay una indicación real( ${ }^{(21)}$.

Existe evidencia de que el dolor crónico tiende a ser infratratado, los factores que contribuyen incluyen aspectos como información insuficiente sobre la fisiología del dolor en el personal de salud, baja sensibilidad y especificidad del diagnóstico, falta de directrices de tratamiento y la mala comprensión de los mecanismos de acción de los analgésicos con la consecuente renuencia a utilizar ciertos agentes farmacológicos. El uso de opiáceos para el dolor no oncológico es controversial debido a algunas consideraciones sobre su eficacia, seguridad y la posibilidad de adicción. Asimismo, las indicaciones del empleo de opiáceos para el tratamiento 
del dolor crónico no oncológico no están bien establecidas. En el estudio de Mainar y colaboradores (2012) se reporta un 20.1\% de pacientes con dolor crónico infratratados donde sólo el 2.3\% recibió opiáceos fuertes ${ }^{(6)}$. Una revisión de Gálvez (2009) describe que sólo el 5\% de los pacientes en Europa recibe tratamiento del dolor crónico con opiáceos fuertes, aunque hubo cierta variabilidad entre los países ${ }^{(22)}$.

En los resultados del presente estudio se revelan cifras aún más altas de infratratamiento, previo a la valoración inicial del CIETD-CP sólo el 18\% de los pacientes había utilizado o se encontraba en tratamiento con algún opiáceo y el $45 \%$ con AINE. Después de la prescripción del tratamiento en el CIETD-CP, esta relación se revirtió con el empleo de opiáceos para el dolor de origen musculoesquelético en el $89 \%$ de los pacientes y sólo en el $6 \%$ se mantuvo la continuidad del AINE.

La base fisiológica del dolor agudo consiste en la estimulación de los nociceptores que desencadenan el proceso de nocicepción, con ello el umbral de estos receptores disminuye y se facilita la respuesta nocifensiva para promover una adecuada recuperación de los tejidos, esto se conoce como sensibilización periférica. Sin embargo, si el dolor agudo no es manejado correctamente existe el riesgo de que se genere una sensibilización central, en donde se produzcan mecanismos neurobioquímicos en el sistema nervioso central que pueden perpetuar el dolor, independientemente de lo que ocurra a nivel periférico ${ }^{(23)}$. Debido a esto, un oportuno y adecuado tratamiento del dolor agudo puede evitar que el dolor se cronifique.

En los resultados se reportó una prevalencia del dolor neuropático asociado al dolor nociceptivo somático en el $41 \%$, constituyendo el principal diagnóstico algológico y el primer objetivo de tratamiento. Esta cifra está por encima de las reportadas en estudios previos, Elzahaf y colaboradores (2016) reportaron una prevalencia de dolor neuropático en pacientes con dolor crónico de $19.7 \%{ }^{(24)}$. A su vez, Cook y Chastain (2001) en una muestra de 374 sujetos reportaron categorías diagnósticas de dolor neuropático en un 29\%(13) .

Nuestra investigación tuvo algunas limitaciones en las que se identifica que sólo se consideraron los tratamientos farmacológicos, dejando de lado las intervenciones no farmacológicas. Por otra parte, sería recomendable realizar un estudio de seguimiento para valorar la eficacia del tratamiento analgésico inicial e incrementar la evidencia del manejo del dolor de origen musculoesquelético.

\section{CONCLUSIONES}

El presente estudio cumplió con el objetivo de describir el perfil demográfico y clínico de los pacientes que acudieron por primera vez a un centro de estudio y tratamiento del dolor en tercer nivel de atención especializado en padecimientos musculoesqueléticos.

El síndrome doloroso nociceptivo somático con componente neuropático se estableció como el diagnóstico de mayor prevalencia. Es necesario realizar mayor investigación de los mecanismos del dolor neuropático en el dolor de origen musculoesquelético. De la misma manera, estudios epidemiológicos podrían promover una mejor comprensión, identificación de factores de riesgo y medidas de prevención, unificación de criterios de diagnóstico y quizá de tratamiento oportuno. Es posible que el tiempo en que los pacientes son remitidos a una clínica del dolor y reciben un tratamiento específico podría modificar la evolución y repercusiones del dolor crónico. Sin embargo, en la actualidad no todos los pacientes pueden ser evaluados por una clínica del dolor en los primeros momentos de la patogenia, a lo que resultaría factible capacitar a los médicos de primer y segundo nivel de atención para el control oportuno del dolor de origen musculoesquelético.

El incremento de la prevalencia del dolor crónico de origen musculoesquelético es reflejo de la transición demográfica en México y del incremento de la esperanza de vida y de la incidencia de las enfermedades crónicas y degenerativas. El sistema de salud pública deberá estar preparado para sustentar la demanda asistencial en lo que respecta al dolor crónico por enfermedad degenerativa que se generará en los años próximos.

\section{REFERENCIAS}

1. Elzahaf RA, Tashani OA, Unsworth BA, Johnson MI. The prevalence of chronic pain with an analysis of countries with a Human Development Index less than 0.9: a systematic review without meta-analysis. Curr Med Res Opin. 2012;28:1221-1229.

2. Covarrubias-Gómez A, Guevara-López U, Gutiérrez-Salmerón C, Betancourt-Sandoval JA, Córdova-Domínguez JA. Epidemiología del dolor crónico en México. Rev Mex Anestesiol. 2010;33:207-213.

3. Treede R, Rief W, Barke A, Aziz Q, Bennett MI, Benoliel R, et al. A classification of chronic pain for ICD-11. Pain. 2015;156:1003-1007.

4. Montero Matamala A, Samper Bernal D, Vidal Fuentes J, Rodríguez Dinten MJ, Jiménez Cosmes L. Estudio para evaluar el perfil del paciente que acude en primera visita a Unidades de Dolor de centros hospitalarios españoles (estudio PANDHORA). Rev Soc Esp Dolor. 2011;18:219-227.
5. Bair MJ, Matthias MS, Ph D, Nyland KA, Huffman MA, Stubbs DL, et al. Barriers and facilitators to chronic pain self- management: a qualitative study of primary care patients with comorbid musculoskeletal pain and depression. Pain Med. 2009;10:1280-1290.

6. Mainar AS, Artieda RN, Morillo JV, Escobar AE. Treatment profiles and costs of patients with chronic pain in the population setting. Clinicoecon Outcomes Res. 2012;4:39-47.

7. Stump PRNAG, Kobayashi R, Campos AW. Low back pain. Rev Dor. 2016;17:63-66.

8. Smart KM, Blake C, Staines A, Doody C. The discriminative validity of "nociceptive," "peripheral neuropathic," and "central sensitization” as mechanisms-based classifications of musculoskeletal pain. Clin J Pain. 2011;27:655-663. 
9. Covarrubias-Gómez A. Lumbalgia: un problema de salud pública. Rev Mex Anestesiol. 2010;35:106-109.

10. Vos T, Flaxman AD, Naghavi M, Lozano R, Michaud C, Ezzati M, et al. Years lived with disability (YLDs) for 1160 sequelae of 289 diseases and injuries 1990-2010: a systematic analysis for the Global burden of Disease Study 2010. Lancet. 2012;380:2163-2196.

11. Lozano R, Gómez-Dantés H, Garrido-Latorre F, Jiménez-Corona A, Campuzano-Rincón JC, Franco-Marina F, et al. La carga de enfermedad, lesiones, factores de riesgo y desafíos para el sistema de salud en México. Salud Publica Mex. 2013;55:580-594.

12. Kuri-Morales PA. La transición en salud y su impacto en la demanda de servicios. Gac Med Mex. 2011;147:451-454.

13. Cook AJ, Chastain DC. The classification of patients with chronic pain (age and sex differences). Pain Res Manag. 2001;6:142-151.

14. Souza I, Vasconcelos AGG, Caumo W, Baptista AF. Resilience profile of patients with chronic pain. Cad Saude Publica. 2017;33:1-11.

15. Casals M, Samper D. Epidemiología, prevalencia y calidad de vida del dolor crónico no oncológico: Estudio ITACA. Rev Soc Esp Dolor. 2004;11:260-269.

16. Dueñas M, Ojeda B, Salazar A, Mico JA, Failde I. A review of chronic pain impact on patients, their social environment and the health care system. J Pain Res. 2016;6:457-467.
17. Nicholas MK. When to refer to a pain clinic. Best Pract Res Clin Rheumatol. 2004;18:613-629.

18. Ortiz-Pereda V, López M, Arroita A, Aguilera L, Azkue J, TorreMollinedo F, et al. Antiinflamatorios no esteroideos y paracetamol en el tratamiento del dolor. Gaceta Médica de Bilbao. 2017;104:148-155.

19. Guevara-López U, Covarrubias-Gómez A, Rodríguez-Cabrera R, Carrasco-Rojas A, Aragón G, Ayón-Villanueva H. Parámetros de práctica para el manejo del dolor en México. Cir Cir. 2007;75:385-407.

20. Beaulieu P, Lussier D, Porreca F, Dickenson A. Pharmacology of pain. Seattle (US): IASP; 2010.

21. Vergne-Salle P. La escalera analgésica de la OMS: ¿es apropiada para el dolor articular? De los AINE a los opioides. Asociación Internacional para el Estudio del Dolor. 2016;18:1-4.

22. Galvez R. Variable use of opioid pharmacotherapy for chronic noncancer pain in Europe: causes and consequences. J Pain Palliat Care Pharmacother. 2009;23:346-356.

23. Ramos-Alaniz A, Guajardo-Rosas J, Chejne-Gómez F, Juárez-Lemis AM, Ayón-Villanueva H. Mecanismos para prevenir dolor agudo a crónico. Rev Mex Anest. 2018;41:S44-S47.

24. Elzahaf RA, Johnson MI, Tashani OA. The epidemiology of chronic pain in Libya: a cross-sectional telephone survey. BMC Public Health. 2016;16:776.نं 